\title{
Interpretation und Bündnis. Anregungen von Klaus Heinrich und Gianni Vattimo für eine nicht-fundamentalistische Re-Interpretation des christlichen Narrativs
}

\author{
Jakob Helmut Deibl
}

This article asks for the genuine resources of the Christian narrative against fundamentalist tendencies within (Christian) religion. It presupposes that critical reflection on religion and reflection within religion cannot be separated from each other: Critique of religions and illumination processes within them must always be combined. In the first chapter I examine three types of fundamentalism according to three main questions highlighted by the philosopher Klaus Heinrich concerning identity, language, and selfdestruction. In the second chapter, I refer to these questions by looking at important motives of Gianni Vattimo's re-interpretation of Christianity. In the last chapter I ask whether the motives picked up in Heinrich and Vattimo can find their centre within the biblical term covenant.

Since 2013 post-doctoral fellow at the department for Systematic Theology at the Faculty of Catholic Theology (University of Vienna); guest lecturer at the Pontificio Ateneo Sant'Anselmo (Rome); member of the research platform "Religion and Transformation in Contemporary Society" (University of Vienna).

\section{Vorweg ...}

Der folgende Beitrag fragt nach Ressourcen, welche die christliche Religion in ihrer Tradition findet, um fundamentalistischen Haltungen, gerade in ihrem eigenen Umfeld, kritisch zu begegnen. Nicht von einem äußerlichen Standpunkt wie etwa dem Gedanken der Toleranz oder der Menschenrechte wird die Argumentation ihren Ausgangspunkt nehmen, sondern von der Logik des christlichen Narrativs selbst, wobei die Motive Bund/Bündnis und Interpretation im Mittelpunkt der Überlegungen stehen werden. Den ersten Terminus werde ich über das Denken des Berliner Philosophen Klaus Heinrich, den zweiten über den Turiner Philosophen Gianni Vattimo aufgreifen. Damit sind auch die beiden wichtigsten Gesprächspartner dieses Artikels benannt. Vor den eigentlichen Überlegungen sind einige Vorbemerkungen nötig.

1) Aufklärung in den Religionen (nach Klaus Heinrich): Hinter der Frage nach den anti-fundamentalistischen Ressourcen innerhalb einer religiösen Tradition steht die Überzeugung, dass in den heute allgegenwärtigen Diskursen über reli- 
giöse Radikalisierung nicht primär die Frage zu stellen ist, wie viel Religion in säkularen Staaten zumutbar oder wünschenswert ist, sondern die Frage, inwiefern Religionen das in ihnen angelegte Potential zu einem kritischen Umgang mit ihren eigenen radikalen Tendenzen nutzen. Die Auseinandersetzung damit, nicht den Rückzug aus dem öffentlichen Raum, können und müssen Staat und Zivilgesellschaft heute den Religionen zumuten. Damit werden Religionen in dem aufklärerischen Projekt, religiöse Fundamentalismen abzuschwächen, nicht als Gegner betrachtet, sondern als Bundes-Genossen (Klaus Heinrich ${ }^{1}$ ).

Diese Überlegung geht von einer Unteilbarkeit der Aufklärung aus, die per se ein offenes, unabschließbares und vor allem nicht partikularisierbares Projekt ist. Wer sich auf den Standpunkt der Aufklärung beruft, muss in der Identifizierung von so genannten unaufgeklärten Wissensformen und der Kritik an primär traditionsgestützten Narrativen offen bleiben für aufklärerische Tendenzen in diesen selbst. Sonst würde das Projekt der Aufklärung regionalisiert, begrenzt und zu einem Identitätsmarker herabgestuft, welcher bloß der Selbstdefinition mittels des eigenen (angemaßten) Wissensstatus dient - nach dem Motto: „Wir sind aufgeklärt, ihr hingegen nicht" ". ${ }^{2}$ Dies bedeutet, dass sich eine klare Scheidung von aufgeklärter Zivilgesellschaft (Wissenschaft, freiem Denken ...) einerseits und traditionsgestütztem Lebensvollzug (Religion, Glaube ...) andererseits nicht als aufgeklärt ansehen lässt. ${ }^{3}$ Schließlich erweist sich eine derartige Trennung auch als Methode zur Gesellschaftsanalyse als unzureichend. Das gesellschaftliche Projekt einer Aufklärung den Religionen gegenüber lässt sich nicht abheben von der Frage nach der Selbstaufklärung der Religionen oder in den Religionen. ${ }^{4}$ In den Worten von Klaus Heinrich: Die Religionskritik ,ist nicht zu trennen von dem Moment der Reflexion in den Religionen selbst, die Reflexion in ihnen und ihnen gegenüber ist eine“"5.

Kein Geringerer als Ludwig Feuerbach, einer der Ahnen moderner Religionskritik, erkannte das aufklärerische Moment der Religionen, beschränkte es jedoch in seinem Geltungsbereich, indem er es lediglich darauf bezog, dass Re-

1 Vgl. Heinrich ${ }^{4} 2002$, S. 20.

2 Kant verwehrt sich in seiner kleinen Schrift Beantwortung der Frage: Was ist Aufklärung? gegen eine Verwendung des Prädikates ,aufgeklärt“ im Sinne einer Identitätsgebung und verweist stattdessen auf einen fortschreitenden Prozess: „Wenn denn nun gefragt wird: Leben wir jetzt in einem aufgeklärten Zeitalter? so ist die Antwort: Nein, aber wohl in einem Zeitalter der Aufklärung.“ (I. Kant 1977, 59 (A 491). Vgl. auch Langthaler 2013.

3 Bereits Hegel zeigte in der Phänomenologie des Geistes luzide, dass sich Glaube und reine Einsicht nicht als zwei einander gegenüberstehende Pole festhalten lassen. Vgl. Hegel 1986, S. 391-431.

4 Hat die erste Aufgabe ihren klassischen Ort in der Philosophie, Religionswissenschaft und den Einzelwissenschaften, so die zweite in der Theologie.

5 Heinrich 2000, S. 8. 
ligionen jeweils die ihnen vorhergehenden religiösen Traditionen als falsches Bewusstsein entlarven. Dass die jeweils aktuelle, mit kritischem Anspruch auftretende Ausformung der Religion als Religion derselben Kritik durch eine später auftretende Ausgestaltung der Religion verfallen müsse, könnten die Religionen nicht sehen. Diese Einsicht komme erst der Philosophie zu. Bezeichnend ist, dass in dieser Betrachtung sowohl für die Religionen als auch für die Philosophie das Moment der auf sich selbst gerichteten Aufklärung entfällt. Auch die Philosophie verbleibt im selben Entwicklungsschema wie die einander ablösenden Gestalten der Religion und richtet die aufklärerische Kritik lediglich auf ihr gegenüberstehende frühere Formen des Bewusstseins. ${ }^{6}$ Demgegenüber wird es in diesem Beitrag um einen Begriff der Aufklärung gehen, der nicht von Kampf und Überwindung ausgeht, sondern Aufklärung als das Bestreben ansieht, der Gesellschaft ,ein Bewusstsein ihrer selbst zu geben“7 . In dieses Projekt müssen auch die Religionen einbezogen werden, sollen nicht Teile der Wirklichkeit gänzlich in einen Bereich des Alogischen abgedrängt werden.

2) Offene Re-Interpretation (nach Gianni Vattimo): Die Suche nach antifundamentalistischen, selbstaufklärerischen Potentialen innerhalb des Christentums vollzieht sich in weiterer Folge in einem Dialog mit Klaus Heinrich und Gianni Vattimo. Es handelt sich dabei nicht in erster Linie um eine systematische Darstellung der philosophischen Grundgedanken der beiden, sondern um einen Beitrag über das Christentum. Vorausgesetzt ist mithin, dass die christliche Religion noch die Beweglichkeit und Lebendigkeit hat, Impulse aus ihrem kulturellen Umfeld - Heinrich und Vattimo sind weder Theologen noch Vertreter einer Kirche $^{8}$ - in produktiver Weise aufzunehmen und sich anzuverwandeln. Theologisch produktive Phasen waren wohl immer diejenigen Zeiten, in welchen Kirche und Theologie eine unbefangene Offenheit gegenüber Philosophie und Kultur im Allgemeinen hatten und es so zu einer offenen Re-Interpretation des Christentums kommen konnte. ${ }^{9}$ Letzteres hat sich durch die Jahrhunderte in nicht

\footnotetext{
6 Vgl. Feuerbach 2005, S. 52-55.

7 Heinrich ${ }^{4} 2002$, S. 35.

8 Vgl. Heinrich 2000, S. 36; Vattimo 1997.

9 Um nur einige Beispiele aus verschiedenen Epochen zu nennen: Weder die Theologie des Paulus noch die des Johannes der Apokalypse wären möglich gewesen, hätten sie nicht in intensiver Weise das sie umgebende kulturelle Klima aufgesogen. Der Höhepunkt der Scholastik sowie deren Zerbrechen im theologischen Nominalismus setzen die von außen kommenden Anstöße der aristotelischen Philosophie voraus. Der Einfluss Teilhard de Chardins auf den theologischen Aufbruch im 20. Jahrhundert hängt wesentlich damit zusammen, dass ein Theologe es vermochte, in produktiver Weise an der naturwissenschaftlichen Forschung seiner Zeit teilzunehmen. Karl Rahner konnte vor dem Hintergrund der Philosophie von Leibniz bis Heidegger seine Theologie entfalten, was Johann Baptist Metz um die Rezeption der neomarxistischen Denker erweiterte.
} 
unerheblichem Maße durch „Kultur“ im weitesten Sinne ausgedrückt, d.h. durch Ausdrucksweisen, die es zwar mitgeprägt hat, aber nie vollständig zu kontrollieren vermochte und auch nicht als „Eigentum“ für sich veranschlagen konnte. ${ }^{10}$ Wo es sich kulturkämpferisch als Gegenkultur stilisiert und seine Disposition zur ReInterpretation verliert, handelt es sich um eine Verfallserscheinung, die nichts mit einer konstruierten Alternative „,konservativ“/,liberal“ zu tun hat, sondern mit der Unfähigkeit kulturelle Prozesse aufzunehmen und $\mathrm{zu}$ amalgieren. ${ }^{11}$ Der Versuch, von ,,außen“ oder von den Rändern kommende Impulse in die Selbstverständigung der Religion aufzunehmen, muss nicht notwendigerweise zu Qualität und kreativen Aufbrüchen führen, verhindert jedoch, dass ein Denken, eine Theologie, ein religiöser Narrativ sich als in sich geschlossen und suffizient ansieht.

Zwei Autoren, die keinen direkten Bezug aufeinander haben, miteinander ins Gespräch zu bringen, ist bereits eine Reminiszenz an einen der beiden Autoren, nämlich an Gianni Vattimo, der selbst in seinen Texten immer wieder sehr unterschiedliche Denker in einen Dialog bringt, sodass sich ein „Zwischen“, ein unerwarteter offener Raum der Re-Interpretation der jeweiligen philosophischen Entwürfe entfalten kann, welcher sich in der akribischen Wiedergabe einer Denkschule nicht eröffnen könnte. ${ }^{12}$ Die sich in einer derartigen Zusammenstellung aufbauende Spannung, die Überschneidungen und gegenseitigen Begrenzungen können - vielleicht - etwas von jenem geistigen, kulturell sedimentierten Hintergrund ans Licht bringen, aus dem verschiedene Formen des Denkens in einer Epoche leben, ohne dass sie diesen Horizont jemals unmittelbar aussprechen könnten.

3) Das Christentum, von dem hier die Rede sein wird, hat (zumindest in den westlichen) Kulturen einen doppelten Verlust erlitten, insofern ihm einerseits die Fragen und andererseits der Mythos abhandengekommen sind, wobei beides in einem engen Zusammenhang steht. Es verkündet - darin greife ich bereits auf Überlegungen Klaus Heinrichs zurück - seine Botschaft primär als Lehre und nicht mehr als Antwort auf Fragen. Heinrich zufolge müssten alle theologischen, philosophischen und mythischen Konzepte immer wieder daraufhin befragt

10 Karlheinz Ruhstorfer bringt sehr schön die grundsätzliche Verwiesenheit von Theologie und Kirche auf die jeweils aktuelle Kultur zum Ausdruck: Um der Aufgabe gewachsen zu sein, in inspirierender Weise auf die eigene Zeit zu wirken, „müssen sich die konkreten Ausprägungen des Christlichen stets durch den Fortschritt der autonomen Kultur unserer Tage hinterfragen und erneuern lassen. Die Verkündigung, die Liturgie und die karitativen Aktivitäten der Kirchen wie auch die Spiritualität jedes Christen und jeder Christin bedürfen der Rückbindung an die Dynamik der Kultur.“ (Vgl. Ruhstorfer 2015, S. 29)

11 Vgl. Appel 2014, 303-320.

12 Vattimo versuchte anfänglich Marx und Nietzsche aufeinander zu beziehen, später Nietzsche und Heidegger, Heidegger und Benjamin, Heidegger und Paulus. 
werden, inwiefern sie Antwort auf eine Bedrohung sind. Wenn es dem Christentum nicht mehr gelingt, die Fragen heutiger Menschen, die vielfach nicht direkt ausgesprochen werden können, hörbar zu machen, verliert es auch seine Qualität als Bündnispartnerin gegen bedrohende Ängste. Seine Botschaft hat dann nicht mehr die Qualität einer Erzählung, in welcher Menschen eine Deutung ihrer Existenz zu lesen vermögen, indem sie ihre Geschichte darin einzuschreiben beginnen (Mythos), sondern verengt sich zu abstrakter Lehre, zu Recht oder Moral. Ist es nicht gerade dieser Niedergang narrativer Kompetenz in den westlichen Kulturen, der nun auch andere Weltgegenden erfasst und als massive Bedrohung empfunden wird, was nicht selten fundamentalistische Reaktionen hervorruft? Wenn dies zutrifft, müsste der Fundamentalismus als Phänomen und Spiegel unserer Zeit verstanden werden und nicht als ein Zurück in eine vormoderne Welt.

4) Der folgende Beitrag umfasst zwei Kapitel, wobei das erste von Klaus Heinrich seinen Ausgangspunkt nimmt, das zweite von Gianni Vattimo. Im ersten Kapitel werden mit Bezug auf drei menschliche Grundfragen, die Klaus Heinrich identifiziert, drei Ausdrucksweisen des Fundamentalismus entwickelt. Im Unterschied zu vielen anderen aktuellen Beiträgen über religiösen Fundamentalismus beziehe ich mich nicht auf Fallbeispiele, sondern versuche ansatzweise eine Typologie der Phänomene zu geben, die meist unhinterfragt mit Fundamentalismus bezeichnet werden. Im zweiten Kapitel werde ich in Antwort auf die zuvor entwickelten Formen des Fundamentalismus mit Gianni Vattimo einige Eckpunkte für ein nicht-fundamentalistisches Verständnis des christlichen Narrativs zu entwickeln suchen. Es folgt ein Nachwort, welches die Überlegungen dieses Beitrags mithilfe der Kategorie des „Bundes“ noch einmal zusammenzufassen sucht.

\section{Kleine Typologie des Fundamentalismus (nach den Grundfragen von Klaus Heinrich)}

Das Denken des Religionsphilosophen Klaus Heinrich ${ }^{13}$ steht der Frankfurter Schule nahe und hat wichtige Ausgangspunkte auch in der Psychoanalyse Freuds, bei Hegel, im griechischen Mythos und im biblischen Denken. Wie ein Leitmotiv kann der folgende Satz gelten: „Wir verstehen“ einen Begriff nur dann, wenn es uns gelingt, den in ihm verkörperten ,Protest' zu übersetzen. ${ }^{\text {“14 }}$ Philosophie ist mithin die Arbeit einer Übersetzung. Bis zu den grundlegenden Sätzen der formalen Logik müssen philosophische, theologische und mythologische Motive als Antworten auf eine Bedrohung gelesen werden. Als Antwortversuche stellen sie

13 Bislang gibt es leider wenig Sekundärliteratur zu Klaus Heinrich. Es sei jedoch verwiesen auf die Diplomarbeit: Stadlbauer 2014 sowie auf Pittl (in Vorbereitung).

14 Heinrich ${ }^{4} 2002$, S. 109. 
ein wenigstens partielles Gelingen in der Begegnung mit der zerstörenden Macht dieser Bedrohung dar. Von hier aus ergibt sich bereits ein wichtiger Hinweis auf die Frage der Aufklärung und Selbstaufklärung der Religionen. Es geht Heinrich nicht - wie es das Anliegen der klassischen Religionskritik war - um ein Entlarven der religiösen Antworten in ihrer Insuffizienz, ihrem Trug- oder Projektionscharakter, sondern um die Frage, inwiefern sie einen Protest gegen eine zersetzende Gefahr darstellen und damit ein emanzipatives Potential verkörpern. Wie weit reicht ihre Macht des Widerstehens und wie können sie als Bundes-Genossen gewonnen werden, auch wenn ihre Begrenztheit eingesehen ist?

Klaus Heinrich zufolge gibt es drei Grundtypen von Fragen, die zu einer Antwort des Protestes gegen eine mit ihnen verbundene Bedrohung auffordern, nämlich die Fragen nach Identität, Sprache und Selbstzerstörung. Diesen korrespondieren ,die Bedrohung, mit nichts identisch zu sein; die Bedrohung, keine Sprache zu haben unter den zerstörenden Mächten; die Bedrohung, daß die Angst des Identitätsverlustes und der Sprachlosigkeit in Selbstzerstörung treibt ${ }^{\text {"15 }}$. Den drei Grundtypen werde ich im Folgenden verschiedene Ausprägungen des Fundamentalismus zuordnen, die sich freilich überschneiden und miteinander verbinden können. ${ }^{16}$

1) Was gibt uns Identität? Klaus Heinrich fragt: „Wo finden wir Modelle einer standhaltenden Identifikation? Alle Religionen entwerfen solche Modelle. Ihre Offenbarungen sind Offenbarungen des Standhaltens, der Leben erhaltenden und Leben erzeugenden Identifikation."17 Angesichts der Bedrohung durch Identitätsverlust trägt die Versicherung eigener Identität die Formel A=A, die für Heinrich mitnichten bloß leere Tautologie ist, sondern Symbol des Widerstehens: Etwas ist mit sich selbst identisch und wird weder von widerstreitenden Mächten zerrissen noch löst es sich in zusammenhangslose Ereignispartikel auf. Die Formel hat dabei ,ein konservatives Moment und ein Moment der in die Zukunft gehenden Utopie“"18. Erstens: A bleibt A und verliert sich nicht in den Veränderungen der Zeit. Zweitens: A erweist sich, tatsächlich A zu sein, verwirklicht das, was es ist. Drittens: Diese beiden divergierenden Momente muss der Satz $A=A$ in der Kopula (dem ,ist“), dem verbindenden Band, der gemeinsamen Sphäre der Vermittlung balancieren. Je besser diese Balance gelingt, desto tragfähiger sind

\section{Heinrich 1992, S. 67.}

16 Klaus Heinrich formuliert die erwähnten drei Fragen nicht im Hinblick auf eine Auseinandersetzung mit unterschiedlichen Formen des Fundamentalismus, sondern verbindet sie mit drei Phasen der abendländischen Geschichte, was hier nicht weiter erläutert werden kann. Es wäre interessant zu fragen, welche Gemeinsamkeiten und Unterschiede sich zu Ruhstorfers Bestimmung von drei Phasen der Geschichte, nämlich einer Bestimmung des metaphysischen Zeitalters von der Idee her, der Moderne vom Referenten und der Postmoderne von der Interpretation her, ergäben.

17 Heinrich ${ }^{4} 2002$, S. 63.

18 Ebd., S. 60. 
der Protest und die Macht des Widerstehens, die sich in der Formel zum Ausdruck bringen. Sämtliche Narrative, die Stabilität und Orientierung gewähren, stellen Klaus Heinrich zufolge eine Verkörperung jener Formel dar, ein ausgezeichneter Ort davon ist die Religion.

Fundamentalismus und Identitätsgebung: Weltweit zeigt sich das Phänomen eines massiven Traditionsabbruches und eines Verlustes des kulturellen Gedächtnisses. Über lange Zeit in identitätsstiftenden Erinnerungen eingeborgene Existenzweisen brechen zusammen, symbolische Ordnungen (d.h. Formen kultureller, sprachlicher, religiöser Vermittlung des Daseins) verdunsten. Fundamentalismus antwortet auf das Schwanken der in der Formel A=A ausgesprochenen Balance, indem er alles Gewicht auf deren ersten Aspekt („A ist, was es immer schon war.") legt, dabei aber die Zukunftsdimension, dass Identität sich in je neuen Verkörperungen verwirklichen muss, aus den Augen verliert. Dass in jedem Urteil $\mathrm{A}=\mathrm{A}$ auch eine Trennung, eine Differenz und damit ein Aufschub waltet, macht er vergessen durch eine übersteigerte Identifikation, d.h., er verliert zusehends das inhaltliche Moment, das zu affirmieren er vorgibt, um rein den Akt vollständiger Identifikation auszustellen. Die jeweiligen Inhalte werden dabei völlig austauschbar, sie sind bloße Marken, virtualisierte Images. ${ }^{19}$ An die Stelle der in der Formel der Identität ausgesprochenen Vermittlung (,A ist A, A muss sich als A erweisen“) tritt ein Kult der Unmittelbarkeit, der zu unverdorbenen Anfängen der religiösen Tradition zurückkehren zu können meint. Ihre Vermittlung durch Geschichte und Kultur vermag er nur als Geschichte des Verfalls zu sehen. ${ }^{20}$ Daraus resultiert auch der fundamentalistische Hass auf Kunst, der in gleicher Weise Stätten uralter Tradition wie zeitgenössische Erscheinungsweisen trifft, insofern es sich dabei um Formen handelt, in denen sich der Prozess kultureller Vermittlung, wie er eine spezifische Epoche prägt, einen Ausdruck gibt. Dieser Eindruck verstärkt sich durch die Beobachtung, dass sich fundamentalistische Bewegungen in hohem Maße offen für die Aufnahme der aktuellen Technik zeigen, ihnen umgekehrt aber jeder Sinn für die aktuelle Kunst fehlt. Während Technik den Charakter kultureller Vermitteltheit in einer von

19 Vgl. Appel 2015, S. 19-60.

20 Um ein Gegenbeispiel jüngeren Datums anzudeuten, sei an dieser Stelle verwiesen auf Karlheinz Ruhstorfers Versuch, eine Re-Narration des Christentums explizit im Horizont des zeitgenössischen kulturellen Umfeldes zu entfalten: „[...] um sich tatsächlich in die je eigene Zeit inkarnieren und verstreuen zu können, muss sich der Glaube vorbehaltlos auf die zeitgenössische Kultur einlassen - im Wissen darum, dass die Welt dem Glauben niemals etwas Fremdes sein kann, wenn der Inhalt des Glaubens darin besteht, dass Gott zur Welt gekommen ist und wenn diese Verweltlichung Gottes auch die Bedingung der Möglichkeit der Säkularisierung der Kultur ist. Die weltliche Kultur des Abendlandes ist ein erwachsen gewordenes Kind des Glaubens, wie andererseits auch das jeweils konkrete Christentum ein Kind der entsprechenden Kultur ist.“ (Ruhstorfer 2015, S. 27) 
gewachsenen Traditionen unabhängigen Universalität zu verbergen sucht, ist Kunst gerade deren explizite Zur-Schau-Stellung. Mit übersteigerter Identifikation und dem Abbruch kultureller Vermittlung hängt auch der Akt, sich einen neuen Namen zu geben, zusammen, wie er bei Leitfiguren fundamentalistischer Gruppen zu beobachten ist.

2) Was gibt uns Sprache? Mit dieser Frage verknüpft Klaus Heinrich eine weitreichende Bestimmung von Sprache: „Nur der hat Sprache, der getrennt ist vom Sein. Nur dort ist Sprache, wo Trennung überwunden ist. Sprache ist Neinsagen zum Nichtsein.“21 Erstens: Sprache erwächst nicht aus dem Sein, als ginge aus dem Nicht-Sprachlichen Sprachliches hervor. Es gibt keinen Übergang vom Sein zur Sprache, als wäre sie ein spätes Produkt evolutiver Entwicklung, das eine ihr vorgelagerte und außerhalb ihrer bestehende seinshafte Wirklichkeit spiegelte, indem sie diese bezeichnet. ${ }^{22}$ Vielmehr setzt sie einen Bruch mit allen Möglichkeiten des Menschen, sich in den Gegenständen seines Weltumgangs, sich im Sein zu reflektieren und sich in diesen Spiegelungen wiederzufinden, voraus. Die Trennung vom Sein ist in eins Erfahrung des Verlustes wie der Freiheit von der im Sein repräsentierten Notwendigkeit. Zweitens: Sprache ist Überwindung der Trennung, insofern sie Fähigkeit zur Übersetzung ist und dafür keine prinzipielle Grenze angegeben werden kann: „Sprechend übersetze ich in einem fort, von einer Sprache in die andere, aber immer: von Sprache in Sprache. “23 Sich der Übersetzung verweigern, bedeutet, die der Sprache zukommende universale Macht der Vereinigung zu partikularisieren und auf einen abgetrennten Bereich, der sich selbst als Ganzes setzt, einzuschränken. Drittens: Indem Sprache Neinsagen zum Nichtsein ist, übersetzt sie nicht nur Sprache in Sprache, sondern auch Nicht-Sprache in Sprache. Sie versteht Sprachlosigkeit nicht als Nicht-Sein, sondern als misslingende Übersetzung, ,die in gelingende übersetzt sein will“24. Dies bedeutet ein sprechendes Verwandeln der Wirklichkeit, eine ,Verwandlung der sprachlichen Wirklichkeit, die diese nicht zerstört‘ ${ }^{\text {25 }}$.

Fundamentalismus und (Ur-)Sprache: Fundamentalismus isoliert, mit Blick auf die obige Bestimmung der Sprache, deren Trennung vom Sein, ohne den Gedanken der Rückkehr in die Gemeinschaft durch Übersetzung zu übernehmen. Er beginnt mit einem Bruch mit bisherigen Ausdrucksformen des Daseins und der Kultur, ohne überzugehen in deren Verwandlung. Auf eine sich in der Gesellschaft immer weiter breit machende Sprachlosigkeit antwortet der Fundamentalismus nicht mit partiell gelingenden Versuchen von deren Übersetzung, d.h., nicht mit einem Erstnehmen der Nicht-Sprache als Sprache, die übersetzt werden

\footnotetext{
21 Heinrich ${ }^{4} 2002$, S. 100.

22 „Es gibt keine Unterscheidung zwischen sprachlicher Wirklichkeit und Wirklichkeit." (Vgl. Ebd., S. 112)

23 Ebd., S. 108.

24 Ebd., S. 116.

25 Ebd., S. 113.
} 
will, sondern mit deren Ersetzung durch die Unmittelbarkeit einer Ur-Sprache. Was erscheinen mag, als erhielten sprachlose Menschen eine Stimme, ist nicht deren eigenes Zur-Sprache-Kommen, sondern deren Beherrschung durch die Heteronomie einer sich als Ur-Sprache darstellenden partikularen Sprache. Ihre bisherige Geschichte vermag sich nicht durch die Aufnahme in eine sie überschreitende Erzählung einer Religion auszusprechen, sondern wird gelöscht, weil Leben in der Ur-Sprache neu geschaffen wird und bisherige Identitäten - wie fragmentarisch auch immer sie waren - in dieser nicht ausdrückbar sind. Menschen wird auf diese Weise eine neue Grammatik aufgeprägt, die fortan ihr Fühlen, Denken und Wahrnehmen strukturiert. Wer in der Ur-Sprache spricht, braucht sich um deren Übersetzung in anderen Sprachen nicht zu kümmern, zumal er ein unbestreitbares Vorrecht hat. Der Vervielfältigung sprachlicher und künstlerischer Ausdrucksformen begegnet man mit einem grundsätzlichen Verdacht. Andere können die Ur-Sprache wohl übernehmen, die unsicheren Übergänge der Übersetzung darf es hingegen nicht geben. Auf diese Weise entstehen starke Distinktionen der Zugehörigkeit zur eigenen Gruppe und des Ausschlusses derer, welche die angemaßte (und dabei immer partikular bleibende) Ur-Sprache nicht annehmen. Fundamentalismus bedeutet einen Ausstieg aus den immer ambivalenten sprachlichen Vermittlungen. Dem Gottes-Namen als höchstem Wort der Sprache, das jeden unmittelbaren Bedeutungs- und Verweischarakter außer Kraft setz ${ }^{26}$, haftet in dieser Konzeption nicht mehr eine Unverfügbarkeit an, vielmehr kann er ob seiner semantischen Leerheit zum alles legitimierenden Machtwort werden.

3) Was bewahrt uns vor Selbstzerstörung? Klaus Heinrichs Analyse dieser Frage stellt zwei Bilder einander gegenüber: Sog und Balance. Jede Form von Selbstzerstörung münde in „die Bewegung des Sogs“ ${ }^{\text {“27 }}$, d.h. in eine Unentrinnbarkeit, die ununterscheidbar werden lässt, ob man sich mit den Anstrengungen, ihr zu entkommen, nicht noch weiter der verschlingenden Bewegung überlasse. Zerstörung wie Selbstzerstörung haben beide dasselbe Ziel des AufgesogenWerdens aller Dinge und schließlich des Selbst von einem schaurig bergenden Nichtsein. Mit dem Ausdruck des Soges radikalisiert Heinrich in gewisser Weise Freuds Analyse des Todes-Triebes, insofern der Sog eine fortgesetzte Bewegung des Sich-Anschließens des Ichs an Mächte, die es ziehen (im Unterschied zum Treibenden des Triebes), ja aufsaugen, ist, die letztlich jede Form von Subjektivität auflöst. Wie bei Freud lässt sich dabei individuelles Geschehen von allgemeinem Zeitgeschehen nicht trennen. Es sind die Ängste einer Epoche, die auch in den Ängsten der Menschen, und zwar jedes Menschen, auftreten. Die Frage ist jedoch, ob eine Balance der Ängste mit der widerstehenden Macht des Mutes gelingt: „Mut, der Angst so balanciert, daß sie so wenig ihn verschlingt wie Nichtsein das Sein [...], braucht Verkörperungen, in denen das Gelingen der

26 Vgl. Rahner 1976, S. 54-56.

27 Heinrich ${ }^{4} 2002$, S. 137. 
Balance vor Augen steht.“28 Derartige Verkörperungen der Balance sind stets fragil, was zu der Frage führt, wie mit ihrem Schwanken oder gar Zerbrechen umgegangen werden kann. Lassen sich die Fragmente einstmals gelingender Verkörperungen übersetzen in Formen neuer Verkörperungen, gelingt es, die Reste in neue Weisen der Bundesgenossenschaft aufzunehmen oder tritt nun die Angst gesteigert hervor als „Angst sich zu verkörpern“ “29 überhaupt? „Angst vor einer Angst, die nicht länger balanciert werden kann (und Balance ist jetzt nur ein anderer Name für Sinn) treibt die Verkörperungen, die sich ihr nicht länger aussetzen wollen, in Selbstzerstörung. “30 Die Angst der Sinnlosigkeit ist Klaus Heinrich zufolge die spezifische Angst unseres Zeitalters. ${ }^{31}$

Die Struktur von Sog und Balance lässt sich auch mit zwei unterschiedlichen Formen der Vereinigung beschreiben, die wie eine Anlehnung an Hegels Jugendschriften wirken ${ }^{32}$ :

Wir kennen zwei Begriffe der Vereinigung. Der eine zielt mit der Beseitigung der Trennung zugleich auf die Beseitigung des Getrennten. Gnade ist Ausgelöschtwerden, sie ist die gelingende Entkörperung. Der andere zielt auf die Überwindung des Trennenden, die das Getrennte auch in der Vereinigung bestehen läßt. Gnade ist die in der Vereinigung des Getrennten gelingende Verkörperung. ${ }^{33}$

Die erste Form der Vereinigung belässt in dieser nichts Haltbares, sie löst jede Verkörperung, die immer auch ein Moment der Scheidung, der Distinktion, der Diskretion hat, auf zugunsten eines Auf- und Angenommen-Werdens durch das Nicht-Sein. Die zweite hingegen stellt sich dar als die Verkörperung der Balance oder Dialektik von Getrenntem und Vereinigtem, Einzelnem und Allgemeinem.

Fundamentalismus und Terror/Radikalisierung: Es gibt einen Fundamentalismus, der aus den vielen Enttäuschungen der zerbrechenden Balance von Angst und Mut entsteht und schließlich in eine Ablehnung jeglicher die Ängste und Herausforderungen balancierenden Verkörperungen mündet. Daraus entsteht eine Faszination für die alles an sich ziehenden Sogwirkungen, wie sie in den heute allgegenwärtigen apokalyptischen Szenarien anschaubar werden. Auszulöschen und ausgelöscht zu werden, kann in gleicher Weise als Gnade erfahren werden, die aus den immer fragilen und scheiternden Verkörperungen von Balance erlöst. Führt diese Dynamik dann schließlich bis zur Inszenierung von bzw. in Attentaten und Selbstmordattentaten, so steht dahinter nicht der heroische Akt als Ausdruck einer Form von Männlichkeit, sondern der feierliche Akt des Opfers und der

\footnotetext{
28 Ebd., S. 138.

29 Ebd., S. 139.

30 Ebd., S. 139.

31 Ebd., S. 140.

32 Für diesen Hinweis danke ich Kurt Appel. Vgl. Appel 2003.

33 Heinrich ${ }^{4} 2002$, S. 152 f.
} 
Weihe an das Nichtsein. Nicht ein verzweifeltes Sich-Aufbäumen von Subjektivität zeigt sich darin, sondern das Streben nach einer Vereinigung mit dem Nichtsein, die jede Subjektivität auflöst. Dabei gibt es noch eine weitere Form der Sogwirkung zu bedenken: Nicht allein als postkoloniale Phänomene oder Antwort auf westliche Formen militärischer Intervention sind die sich rasch ausbreitenden terroristischen Fundamentalismen von heute zu verstehen, sondern auch als Antwort auf die immer umfassender werdende ökologische Krise, welche gegenwärtig als ein Akt der Selbstzerstörung der Menschheit vor unseren Augen inszeniert wird. Die gesamte Menschheit befindet sich im Sog einer anonymen Dynamik der Selbstauslöschung. Der Akt des Terrors ist kein Versuch der revolutionären Befreiung daraus, um eine neue Weltordnung zu begründen, sondern wirft sich aktiv in den Sog hinein und macht dadurch, einem Spiegel gleich, offenbar, auf welcher Straße sich die Menschheit befindet.

Nur vor dem Hintergrund des Motives des Soges lassen sich die erschreckenden Berichte, wie schnell die gezielte Radikalisierung von Selbstmordattentätern vor sich gehen kann, deuten. Wo sich ein Denken ohne Auswege, d.h. ohne die Möglichkeit, sich umzudrehen und wegzugehen, zeigt, ist das ein Hinweis, dass bereits Sogwirkungen aufgetreten sind. In diese wird das Dasein eingewiesen durch das systematische Abschneiden bisheriger Lebensumfelder und Beziehungen (als möglicher Auswege) sowie das Verbot der auflösenden Zerstreuung ${ }^{34}$ der Aufmerksamkeit durch Formen der Kunst und des kulturellen Milieus. Die Suche nach immer wieder neu zu findender Balance wird dabei als Schwäche diskreditiert, die sich einem völligen Sich-Anvertrauen der Gnade des Verschlungenwerdens verweigert. Auf die leitende Frage, was uns vor Selbstzerstörung bewahre, reagiert der Fundamentalismus nicht mit partiellen Versuchen des Widerstehens, sondern damit, dass er Verschlungenwerden in ein AngenommenSein aus Gnade umdeutet. ${ }^{35}$

\section{Re-Narration des Christentums (nach Gianni Vattimo)}

Auch ein faszinierendes Denken kann eine Sogwirkung entfalten, die erst mit der Entdeckung zu Bewusstsein kommt, dass man bereits begonnen hat, die Sprache dieses Denkens zu imitieren. Um sich davor zu bewahren, bedarf es weiterer Bündnis-Partner, weil sich im Dialog mit ihnen andere Perspektiven und Ausgänge eröffnen können. Ich wende mich darum mit einem harten Schnitt Gianni Vattimo zu, freilich ohne die Prägung ablegen zu können und ablegen zu wollen, die der Durchgang durch das Denken Klaus Heinrichs mit sich gebracht hat. Ausgehend vom Motiv des Soges lässt sich eine Brücke zu Vattimos Rekonstruktion des christlichen Narrativs bilden, an dessen Grund er eine Auflösung

34 Vgl. Wenzel (erscheint Herbst 2016) und Benjamin 1997, S. 471-508, hier: S. 504.

35 Vgl. Heinrich ${ }^{4} 2002$, S. 131-155. 
jeglicher metaphysischer Fundamente sieht. Dies zeigt sich nicht zuletzt darin, dass die Letztgültigkeit der die sozialen Ordnungen begründenden Grenzen außer Kraft gesetzt wird, indem es nicht mehr Juden noch Hellenen, Sklaven noch Freie, Mann noch Frau (Gal 3,28) gibt. Diese Bewegung kann in zwei Szenarien münden: erstens in einen alles zersetzenden Nihilismus, dessen Sogwirkungen nach der Auflösung der Sicherheit gewährenden Grenzen und Fundamente einem jede Möglichkeit des Widerstehens gegen Bedrohungen raubt. Der Mensch erfährt sich diesen schutzlos ausgesetzt. Was Klaus Heinrich allgemein als Gefahr des Verschlungenwerdens im Sog expliziert hat, zeigt sich im Christentum als Gefahr eines alles zersetzenden Nihilismus, der so ohne die christliche Botschaft wohl nicht denkbar wäre. ${ }^{36}$ Zweitens aber eröffnet sich die Möglichkeit eines „schwachen Denkens“"37, das im Folgenden zu erläutern ist.

Als Vattimo 1983 gemeinsam mit Pier Aldo Rovatti den Begriff des pensiero debole (schwaches Denken) in die philosophische Diskussion einbrachte, verstand er dieses Denken vor allem als Übernahme des Erbes der Dialektik und der Differenz, wobei erstere mit einer Linie assoziiert ist, die von Hegel bis zu den neomarxistischen Denkern reicht, zweitere hingegen mit Nietzsche und Heidegger. Vattimo übernimmt beide Denkweisen weder in unmittelbarer Affirmation noch im Gestus der Überwindung, sondern greift ihre „dissolutive Tendenz ${ }^{\text {‘38 }}$ der Schwächung, d.h. der Auflösung von metaphysischen Letztbegründungen, auf. Dies führt ihn jedoch nicht allein zu einer Betrachtung der Ereignishaftigkeit der Auflösung starker metaphysischer Begründungsstrukturen, sondern zu einer geschichtsphilosophischen Re-Lektüre abendländischer Geschichte, indem er verschiedene „Prozesse der Schwächung ${ }^{\text {“39 }}$, und zwar in den Bereichen Philosophie, Religion, Politik und Wissenschaft, nachzeichnet. In diesen Prozessen der Schwächung zeige sich die „Substanz der Moderne“40. Eine derartige Lektüre abendländischer Geschichte unter dem „Leitfaden der Schwächung “41 hebt sich einerseits von der Erzählung der europäischen Geschichte als Aufstieg und Siegergeschichte, andererseits von einer Verfallsgeschichte, die Europa in einem Nichts enden lässt, ab. Die Rekonstruktion dieser Geschichte als einer Geschichte des Abschiedes von starken, d.h. metaphysisch Sein und Dasein begründenden Strukturen führt Vattimo zu einer neuen Aufmerksamkeit für das christliche Narrativ. Er sieht das schwache Denken „in ununterbrochener Kontinuität zur christlichen Tradition“ “42, wo diese selbst ihre metaphysischen Fundierungs- und kirchlichen Disziplinierungsversuche aufgibt:

\footnotetext{
36 Vgl. dazu Illich 2006, S. 71-89.

37 Vgl. Vattimo 2000, S. 79-97.

38 Ebd., S. 84.

39 Vattimo 2004, S. 125.

40 Ebd., S. 125.

41 Ebd., S. 126.

42 Ebd., S. 127.
} 
Einen Gang der Geschichte als auf dem Wege über die Aufzehrung der starken Strukturen - des Denkens, des individuellen Bewußtseins, der politischen Macht, der sozialen Bindungen, der Religion selbst - auf die Emanzipation gerichtet denken, wird das nämlich nicht eine Art und Weise sein, von der Geschichtsphilosophie her die christliche Botschaft der Fleischwerdung Gottes, die beim hl. Paulus auch kénosis, also Erniedrigung, Demütigung, Schwächung Gottes heißt, zu übersetzen $?^{43}$

Wie Klaus Heinrich sieht Vattimo mithin Philosophie als eine Aufgabe der Übersetzung an. In den Mittelpunkt der Übersetzungsarbeit Vattimos tritt immer stärker das paulinische Motiv der Inkarnation als kenosis (Phil 2,5-11), in welchem eine Schwächung sämtlicher starker Strukturen angelegt ist: Wenn der göttliche logos (Joh 1,1-17) selbst in Gestalt der kenosis in die Geschichte eingeht, welches absolute Fundament könnte dann noch eine unumschränkte ewige Gültigkeit beanspruchen? Das schwache Denken nimmt gewissermaßen von hier seinen Ausgang und begreift sich als Säkularisierung des christlichen Narrativs, welche diesen nicht auflösen oder überwinden will, sondern als dessen Transkription auftritt, die ihrer Herkunft die Treue hält. Andernfalls bliebe sie einem Fortschrittsdenken verhaftet, das in jedem seiner Entwicklungsschritte ein absolutes Urteil über die Vergangenheit spräche und dabei erneut ein absolutes Fundament des Urteilens voraussetzte. Dem positiv verstandenen, von Vattimo affirmierten Nihilismus des schwachen Denkens liegt somit ein religiöser Text zugrunde, der diesen beseelt. ${ }^{44}$ Philosophie und Theologie fallen für Vattimo nicht zusammen, er erkennt jedoch, dass in ihnen ein und dieselbe Reflexion statthat, weshalb sie nicht zu trennen sind und es auch keine Überlegenheit einer über die andere gibt. Vattimo bleibt nicht bei der Konstatierung dieses Bezuges stehen, sondern fragt danach, was die Konsequenzen für Philosophie und Theologie seien, wenn sie diese Beziehung der Transkription nicht als akzidentell ansehen, sondern als die Weise, in welcher uns Philosophie und Theologie heute begegnen, oder besser als die Weise, in der es uns heute möglich ist, eine Re-Narration der abendländischen Geschichte zu geben. Ein wesentlicher Aspekt für die Religion müsste sein, dass sie ihre Berufung der Schwächung ernst nimmt und als Ressource gegen fundamentalistische Tendenzen innerhalb und außerhalb ihrer selbst begreift. Von hier ausgehend mag es gelingen, einige Momente zu explizieren, wie man mit Vattimos Re-Lektüre des Christentums auf die mit Heinrich entwickelten Typen des Fundamentalismus antworten könnte.

1) Christentum und Identitätsgebung: Vattimos Rekonstruktion abendländischer Geschichte gibt eine Möglichkeit an die Hand, sich als aufgehoben im christlichen Narrativ zu sehen, d. h. in diesem seine eigene Geschichte zu erzählen (bzw. erzählt zu finden) und mithin Identität zu erhalten, ohne die Epoche der Moderne aus religiöser Perspektive ablehnen und ihre Entfaltung lediglich als

43 Ebd., S. 127.

44 Vgl. Vattimo 1997, S. 67 f. 
Verfallsgeschichte ansehen zu müssen. Der Zugehörigkeit zur Moderne, aus der auszusteigen ohnehin ein Phantasma darstellt (ist doch mittlerweile die ganze Welt von ihr durchdrungen), kann aus religiöser Perspektive damit auch ein positiver Sinn gegeben werden. Freilich kann es dabei nicht mehr um ein unkritisches Bewohnen von Moderne und Christentum gehen. Die kenosis verpflichtet die von ihr inspirierte (christliche, abendländische) Tradition zu einer beständigen Revidierung möglicher fundamentalistischer Rückfälle, die immer ein starkes, unerschütterliches Fundament affirmieren, von dem sich die eigene Identität (individuell wie kollektiv) in ungebrochener Weise ableiten lässt. Im Zentrum des christlichen Bekenntnisses findet sich demgegenüber mit der Inkarnation in kenosis ein Imperativ zur Kritik, besser noch zur Hinwegarbeitung oder Übersetzung jeglicher fundamentalistischer Ansinnen.

Die dissolutive Tendenz des schwachen Denkens erfasst auch das Konzept des Subjektes als Identität mit sich selbst. Zunächst betonte Vattimo dabei die auflösenden Motive bei Nietzsche, Freud, Musil, Bloch und in der künstlerischen Avantgarde, dann führte er diese Bewegung ebenfalls bis auf Paulus zurück, den er über Heideggers Lektüre der Briefe an die Gemeinde von Thessaloniki aufnimmt. ${ }^{45}$ Die Warnung vor dem Auftreten des Antichristen in den genannten paulinischen Schriften beziehe sich auf die Versuchung der ,idolatrischen Identifikationen des Messias mit Gestalten [...], die dessen Bedeutung verfälschen “46, was eine Form objektivierenden, repräsentativen, d. h. metaphysischen Denkens sei. Mit der Unmöglichkeit der Repräsentation des wiederkommenden Messias (parousia) geht eine Schwächung des glaubenden und ihn erharrenden Subjektes einher. Dessen Konzeption vermag sich nicht mehr in einer Identität mit sich selbst zu schließen, sondern erleidet eine Offenheit, die bei Paulus als thlipsis (Drangsal) bezeichnet wird, ,die das christliche Leben kennzeichnen soll“47 und Vattimo zufolge verstanden werden muss als ,Wachsamkeit, die auf die Parusie gerade weil sie [...] nicht als ein ,objektiv' verkündigtes Faktum begriffen wird lauert wie auf den ,Dieb in der Nacht` [1 Thess 5,2]“48. Die Erfahrung des paulinischen Christen sei zu charakterisieren ,als beständige Verweigerung, sich für ,gesinnt ${ }^{6}[\ldots]$ (soll heißen: sinnvoll $[\ldots]$ ) zu halten, eben als in Erwartung der Parusie erduldete Schwachheit ${ }^{\text {‘49 }}$. In der Erwartung der Parusie als eines nicht repräsentierbaren Ereignisses, vollzieht sich ein Ablassen vom Anspruch der Kategorie des Sinnes und damit der Erwartung, das Dasein als eine sinnvolle Ganzheit zu interpretieren. Der darin sich zeigende Nihilismus führe jedoch gerade nicht in die Sogwirkungen eines positivierten Nichts, sondern in eine gesteigerte Verantwortung: „Es gibt kein ,Modell‘ des christlichen Lebens alter-

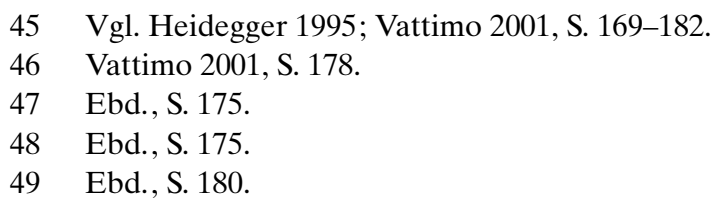


nativ zu jenem, das die geschichtlichen und stets kontingenten Verpflichtungen jedem Gläubigen auferlegen; so wie es auch kein arkanes Wissen der Mysterien gibt, die den Nichtgläubigen verborgen wären. ${ }^{\text {(50 }}$ Damit ist ein Wie? der Existenz angezeigt, das deren inhaltliche Bestimmung, ihr Was?, außer Kraft setzt. Dieser Widerruf jeder objektiven Repräsentation des Daseins findet sich bei Paulus am deutlichsten in 1 Kor 7,29-31, wo er durch das griechische Wort „hos mé“ (,,als ob nicht") ausgedrückt wird:

Dies aber sage ich, Brüder: Die Zeit ist zusammengedrängt; im übrigen, daß auch die Frauen Habenden wie nicht Habende seien und die Weinenden wie nicht Weinende und die sich Freuenden wie nicht sich Freuende und die Kaufenden wie nicht Besitzende und die Gebrauchenden die Welt wie nicht Verbrauchende; denn es vergeht die Gestalt dieser Welt. ${ }^{51}$

Eine Definition der christlichen Existenz kann nicht gegeben werden, weil jeder inhaltlichen Bestimmung die Letztgültigkeit genommen wird. Gleichwohl werden, wie die Wendung des ,als ob nicht“" anzeigt, die inhaltlichen Momente nicht einfach negiert im Sinne ihrer Zerstörung. Gebrochen wird damit vielmehr ein absoluter Ort, von dem aus ein definitives Urteil über die Existenz gesprochen werden könnte (,Etwas ist A.“, „Etwas ist nicht A.“). Das hos mé hält die Existenz in einer Schwebe aus Affirmation und Distanz, die es zu bewohnen gilt und für die - mit Klaus Heinrich gesprochen - immer neue Verkörperungen zu finden sind. Vattimo zufolge gibt das Christentum keine unmittelbare Antwort auf die Frage „Was gibt uns Identität?", schon gar keine fundamentalistische. Es erleichtert vielmehr den hypertrophen Anspruch nach einer fixierten Identität, der uns immer zu groß bleiben muss und verschiebt die Frage nach der Identität zur Frage, wie sich die Fragmente unserer Identität erzählen lassen: Wie können die Übergänge des hos mé zur Sprache kommen, d.h. der Übergang vom Weinen zum Nicht-Weinen etc.? Wie erhält der Zwischenraum, den das hos mé eröffnet, eine Sprache?

2) Christentum und Sprache: Das schwache Denken versteht sich nicht als origineller philosophischer Neuentwurf, sondern als Wiederaufnahme eines Erbes in seinem (für die aktuelle Situation) subversiven Potential. Dieser subversive Rückbezug auf die Erbschaft der Tradition nimmt Abschied von der Anmaßung, die Vergangenheit könne einem abschließenden Urteil unterzogen werden. Dahinter zeigt sich für Vattimo eine ontologische „Struktur“: Für ein Denken des Seins bedeutet dies, dass Letzteres immer "Gegenstand“ einer ReLektüre der Überlieferung ist, in der es sich uns geben, uns zuschicken kann. Es ist nicht das, was (gegenwärtig) ist, vergegenwärtigt werden und als Grund des Seienden verstanden werden kann, sondern als ,schon ,gegangen“ [in seinen sub-

50 Ebd., S. 180.

51 Übersetzung: Münchener Neues Testament. 
versiven Momenten] erinnert" ${ }^{\text {52 }}$ wird: „Dem Sein nähert man sich nicht in der Gegenwart, sondern nur in der Erinnerung, weil (oder einfach: und das bedeutet, daß) es sich nie bestimmt als das, was vorhanden ist, sondern nur als das, was sich überliefert. Sein ist Sendung, Geschick. ${ }^{\text {“53 }}$ Dies bedeutet einen Übergang von einer Metaphysik der Präsenz zu einer hermeneutischen Ontologie. Wir verfügen über keine anderen Kategorien als die uns von der Metaphysik vererbten (und können diese nicht durch neue ablösen), müssen ihnen aber den Anspruch nehmen, die ,stabile Struktur“ ${ }^{\text {654 }}$ des Seins zu spiegeln. Stattdessen sind sie zu begreifen ,als Monumente, als Erbschaft, der gegenüber man pietas empfindet, die man allen Spuren dessen, was gelebt hat, schuldet ${ }^{\text {"55 }}$. Denken bedeutet folglich Andenken und sich in eine Überlieferungsgeschichte zu stellen, außerhalb derer man kein Wort zu sagen vermöchte, die einen aber gleichwohl nicht determiniert. Im Andenken re-konfiguriert sich die niemals objektiv vorliegende Erbschaft der Vergangenheit. Vattimo verwendet dafür den Begriff der Interpretation.

Die eben geschilderte Struktur erweist sich für Vattimo als Erbe der christlichen Erzählung. Die Geschichte der Interpretation stehe in einer Beziehung zu der Geschichte des Heils, wobei die Spannung der beiden Motive, ihr Übergang, ihr Gleiten ineinander aufrechterhalten werden müsse, ohne die Beziehung nach einer Seite hin aufzulösen, indem die Interpretationsgeschichte entweder als Derivat der Heilsgeschichte oder aber als deren Überwindung angesehen würde. Vattimo fasst ihre Beziehung im Wort: „Die Heilsgeschichte geschieht oder ereignet sich nur als Geschichte der Interpretation. ${ }^{\text {"56 }}$ Die Heilsgeschichte führe nicht einlinig zu einem bestimmbaren oder sich entziehenden telos, sondern in eine Vervielfältigung je kontingenter Geschichten. Die christliche Erzählung meint den Abschied vom Phantasma einer letzten großen Erzählung, einer Utopie hin zur Heterotopie, d. h. der Öffnung auf eine Mannigfaltigkeit der kontingenten Geschichten. ${ }^{57}$ Zeichen dafür sei der ,Paracletus, der Geist“, die „,hermeneutische" Person der Dreifaltigkeit ${ }^{\text {“58 }}$, womit Vattimo zufolge die Dimension der Interpretation ins Göttliche selbst hineinreiche. Damit muss die Interpretationsgeschichte (auch dort, wo sie sich im säkularen Bereich ereignet) nicht bloß als Verfallsgeschichte eines Ursprungs, einer Ur-Wahrheit, einer Ur-Sprache betrachtet werden, sondern gibt einer „Produktivität des interpretatorischen

52 Hier zeigt sich einerseits der Einfluss Heideggers (vgl. besonders Heidegger ${ }^{2}$ 1976) und andererseits Hegels 1986, S. 88.

53 Vattimo 2000, S. 90.

54 Ebd., S. 90.

55 Ebd., S. 91.

56 Vattimo 2003, S. 155.

57 Der Ausdruck der Heterotopie wird von Vattimo anders als von Foucault verwendet. (Vgl. Vattimo 1992, S. 85-100).

58 Vattimo 2003, S. 157. 
Aktes“59 Ausdruck. Wenn die Inkarnation die Auflösung jegliches absoluten Fundamentes ist und die Geistsendung die Öffnung dieses Ereignisses auf immer neue Weisen seiner Verkörperung, so ist Interpretation dem „Wesen“ der Botschaft gegenüber nichts Nachgeordnetes, sondern ihm koexistent. Sie leitet sich nicht von einem fixierten Ursprung ab, sondern ist an-archisch, gleichursprünglich mit dem Wesen. ${ }^{60}$ Die hermeneutische Ontologie stellt die philosophische Thematisierung dessen dar. ${ }^{61}$

Der Rückgang zu einer „Ursprache“, wie ihn die zweite der erwähnten fundamentalistischen Haltungen insinuiert, ist weder aus christlicher Sicht noch aus Sicht der Moderne möglich. Es wäre die Affirmation eines Ursprungs, in dessen Nähe man sich zu halten vermag, ohne je in den Horizont der Interpretation einzugehen. Wir sind jedoch immer schon in sprachliche Vermittlungen gestellt, die den Charakter der Interpretation haben und sich in jeder Bezugnahme auf sie wieder neu konfigurieren. Eine unvermittelte Sprache außerhalb der Prozesse der geschichtlich kontingenten Interpretationen gibt es nicht.

Die Frage, was lässt uns zur Sprache kommen? verweist aus christlicher Sicht auf den Überlieferungszusammenhang, in welchem wir stehen und von dem wir uns in jedem sprachlichen Akt abstoßen, um im Verlauten der Rede wieder in ihn zurückzukehren und ihn damit verändert zu haben. Vattimos antifundamentalistische Vision ließe sich vielleicht so beschreiben: Unsere Geschichte erzählt sich niemals als Geschichte ungebrochener Identität in einem Überlieferungszusammenhang, sondern ist die „Oszillation" 62 zwischen Affirmation und deren Widerrufung im hos mé, zwischen Heimat und „Un-heimlicheit“63. Aufgrund des produktiven Charakters jedes interpretatorischen Aktes, der nicht allein Neues $\mathrm{zu}$ einem Bestand hinzufügt, sondern vor allem verhindert, dass sich das Ich jemals in sich selbst zu einer Ganzheit verschließt, ist sie auch die Neuschreibung des gesamten Traditionszusammenhangs, wobei die Deutungen als je kontingente selbst niemals eine Absolutheit behaupten können, widerspräche diese doch dem Ausgangspunkt in der durch die Menschwerdung ausgelösten dissolutiven Tendenz.

Spezifisch christlicher (katholischer) Fundamentalismus will gerade die Produktivität des interpretatorischen Aktes und die stetige Neuschreibung des Überlieferungszusammenhanges abhalten. ${ }^{64} \mathrm{Er}$ äußert sich besonders durch die systematische Denunziation theologischer Kreativität als Häresie und etabliert damit eine Art privaten Lehr- und Wächteramtes. Vielleicht hat der Hass des

59 Ebd., S. 160.

60 Vgl. dazu auch Agamben 2010, S. 76-78.

61 Vgl. Vattimo 2003, S. 165.

62 Vgl. Vattimo 1992, S. 65-84.

63 Vgl. ebd., S. 73.

64 Für den Islam müsste die Frage der Produktivität von Erzählung und Text in anderer Weise gestellt werden. 
Fundamentalismus gegen zeitgenössische Kunst auch damit zu tun, dass diese wie Vattimo ausführt - nicht etwas darstellt, sondern durch ihren nicht mehr in klassische Kategorien einzuordnenden Charakter die Koordinaten unserer Wahrnehmung hinterfragt und zu einem neuen Weltentwurf wird. ${ }^{65}$ Der Fundamentalismus bietet dieser Öffnung auf Neues gegenüber in sprachlicher Hinsicht einen in sich geschlossenen Kreislauf der standardisierten, „generalisierten Kommunikation" ${ }^{\star 66}$, wie ihn Vattimo der technisierten Moderne unterstellt, als deren Symbol das Perpetuum mobile oder der Algorithmus fungieren könnte. ${ }^{67}$ In dieser Hinsicht erweist sich der Fundamentalismus gerade nicht als vormodernes Phänomen, sondern als Produkt der Moderne. Demgegenüber sieht Vattimo in einem sprachlichen, d.h. sich in den interpretatorischen Überlieferungszusammenhang stellenden Weltumgang eine konstitutive Ambiguität der Sprache, Ausdrucksformen und Erzählungen, die nicht einen vorübergehenden, wieder auf Eindeutigkeit zielenden Charakter hat. Wo sie angenommen wird, kann sie gerade zum Ort der Freiheit werden:

Die Erfahrung der Ambiguität ist dagegen für die Kunst [und für die Religion] wie die Oszillation und die Un-heimlichkeit konstitutiv; es sind die einzigen Wege, über die sich in der Welt der generalisierten Kommunikation die Kunst [und die Religion] (nicht: noch, sondern vielleicht: endlich) als Kreativität und Freiheit darstellen kann. ${ }^{68}$

Was aber verhindert, dass die anarchischen Momente, wie sie sich in den Begriffen der Interpretation, Oszillation und Ambiguität auch zeigen, nicht am Ende in die Sogwirkungen eines jeden Bedeutungsgehalt zersetzenden Nihilismus münden?

3) Die bisher mit Vattimo herausgearbeiteten Motive der christlichen Tradition, die gegen Fundamentalismus aufgeboten werden können, vermögen in ihrer dissolutiven Tendenz die vielleicht bedrohlichste Form des Fundamentalismus, nämlich die mit der verschlingenden Dynamik von Sogwirkungen einhergehende, nicht zu verhindern, ja scheinen Letztere möglicherweise sogar zu befördern. Christentum als Religion der Inkarnation ist für Vattimo Nihilismus - doch stellt sich die Frage, wie dieser zu deuten ist.

Vattimo unterscheidet mit Nietzsche (und in dessen Gefolge Heidegger) zwei Formen des Nihilismus: Eine Form führe als „Abschluss der Geschichte“ zu einem positivierten Nichts als objektiv entfalteter, metaphysischer Präsenz. ${ }^{69}$ Diese stellt den letzten starken Entwurf von Geschichte auf ein telos hin dar, das dem sich ihm Anvertrauenden mit der Gnade des Ausgelöscht-Werdens ant-

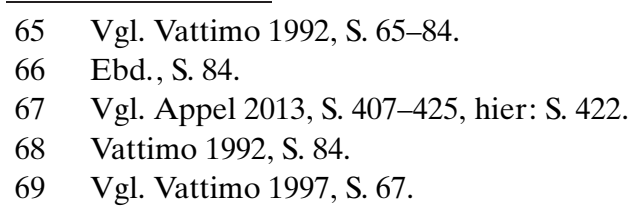


wortet. Dieser Gedanke durchzieht freilich als Erlösungsmotiv latent auch (metaphysische) Formen der Soteriologie, wenn sie all die kontingenten und ambivalenten Geschichten menschlichen Daseins in ein telos absoluter Präsenz in der Ewigkeit des nunc stans münden lassen und sie damit in ihrer Endlichkeit nicht wahrzunehmen vermögen. Es ist nicht völlig abwegig, dass heute, wo derartige Konzeptionen der Erlösung ihre Plausibilität verloren haben, die in ihnen angelegte nihilistische Struktur der Entwertung des Kontingenten im ewigen Ziel der Geschichte frei flottiert und schließlich in der Gestalt eines terroristischen Fundamentalismus, der sich dem letzten Ziel der Vernichtung alles Ambivalenten verschreibt, wieder auftritt. Das Christentum ist in dieser Hinsicht höchst ambivalent, denn Vattimo zufolge ist es auch ein Erbe des Christentums, dass sich in der Geschichte der Philosophie eine kritische Tendenz des Abschiednehmens von der Vorstellung eines letzten alles in sich befassenden Zielpunktes (und sei es in der Gestalt des positivierten Nichts) vollzieht.

Die andere Form des Nihilismus ist für Vattimo eine unendliche (d.h. nicht in das telos eines positivierten Nichts mündende) Geschichte der Schwächung starker Strukturen und des immer neuen Abschiednehmens von Bildern, die sich als nicht fähig erwiesen haben, Repräsentationen des Ichs, der Welt und Gottes darzustellen. Mit der Haltung der pietas müsse man, wie Vattimo bereits zu Beginn der Formulierung des pensiero debole festhält, den Resten und den Trümmern dieser Bewegung der Entwertung begegnen. In der Zuwendung zum christlichen Narrativ entdeckt Vattimo dann das Motiv der caritas (vgl. 1 Kor 13), welches auch in der pietas waltet. ${ }^{70}$ Sie markiert zunächst eine Grenze innerhalb der Auflösung metaphysischer Fundamente, sodass diese nicht in verschlingende Sogwirkungen münde. Wolle man der Schwächung sämtlicher Strukturen treu bleiben, könne es kein äußerliches Prinzip einer Grenze geben, wohingegen sich die Liebe letztendlich als innerstes Moment der Dynamik der Schwächung zeige und darum eine (vielleicht die einzige) nicht gewaltsame Grenze sein könne. Zusammenfassend hält Vattimo fest:

Formuliert man die Idee des Nihilismus als unendliche Geschichte in der Sprache des religiösen ,Textes', der [...] ihm zugrunde liegt und ihn beseelt, redet dieser Text zu uns von der kenosis als direkter und daher auch durch die Liebe Gottes begrenzter und mit Sinn begabter Erfahrung. ${ }^{71}$

Die Prozesse der Schwächung müssen von einem sympathetischen Weltumgang der Liebe geprägt sein; wo andere Motive und Tendenzen leitend sind, bedürfen diese selbst einer Schwächung. Dadurch unterscheidet sich diese Gestalt des Nihilismus auch grundlegend von der alles auflösenden Macht des Skeptizismus.

70 Vgl. ebd., S. 65-69. Zur Wiederentdeckung der Liebe/Agape/caritas an der Schwelle von Philosophie und Theologie vgl. Guanzini (erscheint 2016).

71 Ebd., S. 68. 
Die Schwächung, wie Vattimo selbst sie versteht und in seiner Philosophie praktiziert, vollzieht sich nicht zuletzt als eine Erleichterung des ,apokalyptischen Anteils“ in prägenden abendländischen Konzepten und Begriffen. Im Hinblick auf Nietzsche bedeutet dies eine Re-Lektüre des Übermenschen als des Menschen des über, der - im Abschied von fixierten Bedeutungen - sich in das offene Spiel der Über-gänge von Bedeutungen (d.h. der Interpretationen) stellt und damit jegliche Konnotation des Gewaltmenschen verliert. Heidegger betreffend interpretiert er den alle Ordnungen umstürzenden (d.h. apokalyptischen) Charakter des Ereignisses im Sinne einer Einweisung in die Kontinuität der Überlieferung, in welcher sich Bedeutungen nicht als fixierte, sondern als sich ereignende zeigen.

Die Theologumena der Menschwerdung und der Geistsendung stoßen die Tendenz der Schwächung nicht nur an, sondern beziehen diese auf die christliche Religion selbst zurück, die deshalb ebenfalls einem beständigen Prozess der ReLektüre unterworfen ist. Dabei muss es wesentlich um eine Erleichterung jener apokalyptischen Anteile gehen, die - wenn ihre Partikularität universalisiert wird - in Sogwirkungen zu münden drohen, wie etwa die Motive eines Endkampfes zwischen Gut und Böse oder eines göttlichen Gerichtes, welches alle „,Halbgläubigen“" ${ }^{72}$ vernichten müsse. Stattdessen geht es um neue Bündnisse, die zu schließen sind gegen apokalyptische Sogszenarien.

\section{Zusammenfassung im Bund (nach Klaus Heinrich)}

Wie lässt sich das bisher Gesagte zusammenfassen? Eine von den frühen Mythologien bis $\mathrm{zu}$ den Wissenschaften gängige Methode ist die Integration von Vereinzeltem in ein logisch-genealogisches System. ${ }^{73}$ Von einem Ursprungsprinzip ausgehend wird eine mit logischer Notwendigkeit sich fortsetzende Kette auseinander entspringender Glieder aufgezählt (A ist die Ursache von B, B ist die Ursache von C ...). Vermittels der ununterbrochenen Genealogie wird die Macht der Ursprünge auf die räumlich und zeitlich weit entfernten Glieder übertragen: „Die Funktion der Genealogie [...] ist es, die Macht der heiligen Ursprünge zu übertragen auf das von ihnen Abstammende, aus ihnen Abgeleitete." 74 Die Einbindung des Vereinzelten in ein genealogisches System vermag Identität zu stiften und ihm eine Sprache zu geben, bedeutet aber ein Aufgeben von Freiheit und die Deklaration der aktuellen Lebenswelt zur „Ersatzwelt“"75, welche gegenüber den Ursprüngen immer nur degenerierten, abgeleiteten Charakter haben kann. Im Fall dieses Beitrages würde diese Zugangsweise bedeuten, die

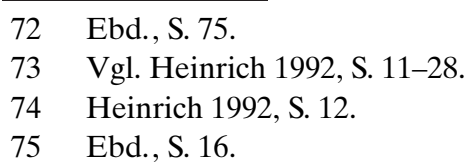


entwickelten Typen religiöser Fundamentalismen und die auf sie Bezug nehmenden Antwortfragmente ausgehend von einem ersten Prinzip nun in eine folgerichtige Ordnung zu bringen. Über dem Gekünstelten eines solchen Versuches verlören die einzelnen Momente überdies die Flexibilität ihrer Anwendung auf gesellschaftliche Prozesse, wie wir sie heute erleben.

Ursprungsmythischen und genealogisierenden Konzepten stellt Klaus Heinrich die biblische Kategorie des Bundes entgegen - oder besser an die Seite. ${ }^{76}$ Der Bund fasst zusammen, indem er Getrenntes verbindet, ohne dessen Freiheit als Preis zu fordern. ${ }^{77}$ Er vermag sich auch auf das zu beziehen, was sich in keine genealogische Beziehung bringen lässt und dem, weil es nicht an der Ursprungsmacht Anteil hat, das Sein abgesprochen werden müsste. So möchte ich abschließend die Frage stellen, ob nicht in all den in diesem Beitrag angestellten Überlegungen, welche ja bewusst von der biblischen Tradition ihren Ausgang genommen haben, die Kategorie des Bundes leitend war.

1) Bund ist kein partikuläres Phänomen: „Zur Dialektik des Bundesgedankens gehört, daß der universale Bund gerade nicht die Ausweitung eines speziellen Bundes ist" ${ }^{678}$, sondern umgekehrt ist die Beschränkung des Bundes auf einen besonderen Bereich die Verzerrung von dessen universalem Anspruch. Hier zeigt sich ein Zusammenhang zum Begriff der Aufklärung vom Anfang dieses Beitrages - letztere ist das Bündnis der aufklärerischen Elemente, wo auch immer sie sich finden (sei es in der Philosophie, der Wissenschaft, dem Mythos, der Kunst, der Religion ...), weil Aufklärung ansonsten partikularisiert würde und gewisse Bereiche der Wirklichkeit von ihr abgetrennt werden müssten.

Für die christliche Religion bedeutet die Universalität des Bundes den Auftrag, Bundesgenossen auch außerhalb ihrer Gemeinschaft zu suchen, um sich in immer neu zu findenden Bündnissen selbst re-interpretieren zu können. Christliche Religion bleibt beständig auf säkulare Philosophie, Wissenschaft und Kunst angewiesen und muss heute lernen, was eine Bundesgenossenschaft mit den auf den Koran aufbauenden Traditionen bedeuten könnte. In diesem Beitrag habe ich den Versuch unternommen, eine Bundesgemeinschaft mit den beiden säkularen Philosophen Klaus Heinrich und Gianni Vattimo zu bedenken. Mit seinen scharfen Distinktionen der Zugehörigkeit und des Ausschlusses sowie der Profilschärfung kann umgekehrt Fundamentalismus zwar eine Form starker Identität geben, dies aber nur um den Preis der Verzerrung des Bundesgedankens, insofern man dessen Geltungsbereich mit der Zugehörigkeit zur eigenen Gruppe zusammenfallen lässt.

2) Das Gegenbild zum Bund ist Verrat. Biblisch geht es nicht um ein Sein, das unberührt vom Verrat ist, sondern um ,ein Sein, das den Mut gibt, trotz des

76 Vgl. Heinrich 1992, S. 61-128.

77 Vgl. Heinrich ${ }^{4} 2002$, S. 152 f.

78 Heinrich 1992, S. 116. 
Verrates den Kampf zu bestehen gegen den Verrat“"79, und zwar „in einer zweideutigen Wirklichkeit unter der Drohung des Verrates“80. Die Proklamation einer Ur-Sprache, einer Utopie, eines telos, in das alle Geschichten zusammengingen, kündigt den Bund mit der zweideutigen Wirklichkeit auf. Sie aus der Gemeinschaft der Sprache nicht auszuschließen und für sie einen Ausdruck zu finden, in ihr mithin nicht nur eine Geschichte des Verfalls zu sehen, ist ein Versuch, der sich in der Betonung von Ambiguität, Oszillation und Heterotopie zeigt.

Die Auflösung eines Denkens, das die zweideutige Wirklichkeit auf ein starkes Fundament bzw. auf Ursprungsmächte zurückführt, öffnet den Blick für die Interpretation und die mit ihr verbundene Produktivität. Aber setzt das dadurch in Gang gebrachte Spiel von Ruf und Antwort, von Übernahme und Fortschreiben eines Erbes, von Korrekturen, Re-Figurationen und Re-Lektüren nicht auch ein Denken des Bündnisses voraus, d. h. eine lose verbundene Gemeinschaft („Kirche als ,Bezugs'-Gemeinschaft für die Gültigkeit und Kontinuität der Geschichte der Interpretation“"81) als Resonanzraum dieses Geschehens?

3) Fundamentalisten, die Identität und Sprache aus der fundamentalistischen Haltung zehren, nicht auf diese ihre Haltung zu reduzieren, heißt, sie als mögliche künftige Bündnispartner ernst nehmen und auch ihnen eine Veränderung ihres Weltzugangs zutrauen. Dies scheint aus meiner Sicht die einzig mögliche langfristige Strategie gegen die diversen, immer wieder sich erneuernden Fundamentalismen und ihre Sogwirkungen zu sein.

\section{Literaturverzeichnis}

Agamben, Giorgio: Herrschaft und Herrlichkeit. Zur theologischen Genealogie von Ökonomie und Regierung (Homo sacer II.2), Frankfurt am Main 2010.

Appel, Kurt: „Gott-Mensch-Zeit. Geschichtsphilosophisch-theologische Erwägungen zu Christentum und Neuem Humanismus im Ausgang von Bibel, Hegel und Musil“, in: Appel, Kurt (Hg.): Preis der Sterblichkeit. Christentum und Neuer Humanismus (QD 271), Freiburg im Breisgau 2015.

Appel, Kurt: „Papst Franziskus, die Vorgängerpontifikate und der Beginn einer neuen symbolischen Ordnung der Katholischen Kirche“, in: Bucher, R. (Hg.): Nach der Macht. Zur Lage der Kirche in Österreich (Reihe „Theologie im kulturellen Dialog“, Band 30), Innsbruck 2014.

Appel, Kurt: „Theologische Erwägungen zum Thema ,Zeit‘ im Spannungsfeld von evolutionärer Weltsicht und biblischer Schöpfungserzählung “, in: Langthaler, Rudolf (Hg.): Evolution und Schöpfungsglauben, Göttingen 2013, 407-425.

$\begin{array}{ll}79 & \text { Ebd., S. } 117 . \\ 80 & \text { Ebd., S. } 117 \mathrm{f} . \\ 81 & \text { Vattimo 2003, S. } 166 .\end{array}$


Appel, Kurt: Entsprechung im Wider-Spruch. Eine Auseinandersetzung mit dem Offenbarungsbegriff der politischen Theologie des jungen Hegel (Religion - Geschichte Gesellschaft. Fundamentaltheologische Studien, Band 31), Münster 2003.

Benjamin, Walter: „Das Kunstwerk im Zeitalter seiner technischen Reproduzierbarkeit“", in: Benjamin, Walter: Gesammelte Schriften. Band I.2, hrsg. von Tiedemann, Rolf / Schweppenhäuser, Hermann, Frankfurt am Main 1997, 471-508.

Feuerbach, Ludwig: Das Wesen des Christentums, Stuttgart 2005.

Guanzini, Isabella: „Die Messianität der Agape. Philosophische und theologische Überlegungen über die Erfüllung der Zeit“, in: Dirscherl, Erwin / Appel, Kurt (Hg.): Das Testament der Zeit (QD 278), in: Appel, Kurt / Deibl, Jakob (Hg.): Barmherzigkeit und zärtliche Liebe. Das theologische Programm von Papst Franziskus. Freiburg im Breisgau (erscheint im Juli 2016).

Hegel, G.W.F.: Phänomenologie des Geistes (Werke 3), Frankfurt am Main 1986.

Heidegger, Martin: Phänomenologie des religiösen Lebens (GA Band 60), Frankfurt am Main 1995.

Heidegger, Martin: „Zeit und Sein“, in: Heidegger, Martin: Zur Sache des Denkens, Tübingen ${ }^{2} 1976$.

Heinrich, Klaus: Versuch über die Schwierigkeit nein zu sagen, Frankfurt am Main / Basel ${ }^{4} 2002$.

Heinrich, Klaus: Vom Bündnis denken. Religionsphilosophie (Dahlemer Vorlesungen, Band 4), Frankfurt am Main / Basel 2000.

Heinrich, Klaus: Parmenides und Jona: vier Studien über das Verhältnis von Philosophie und Mythologie, Frankfurt am Main / Basel, 1992.

Illich, Ivan: In den Flüssen nördlich der Zukunft. Letzte Gespräche über Religion und Gesellschaft mit David Cayley, München 2006.

Kant, Immanuel: Schriften zur Anthropologie, Geschichtsphilosophie, Politik und Pädagogik1 (Werkausgabe Band XI), hrsg. von Wilhelm Weischedel, Frankfurt am Main 1977.

Langthaler, Rudolf: Die Religionsverächter, in: der standard, 10. April 2013, verfügbar unter: http://derstandard.at/1363707572511/Die-Religionsveraechter [07.02.2016].

Pittl, Sebastian: „Alma mater pauperum? Ein fiktives Gespräch zwischen Berlin und El Salvador zur gesellschaftlichen Verantwortung der Universität“, in: Gmainer-Pranzl, Franz / Lassak, Sandra / Weiler, Birgit (Hg.): Theologie der Befreiung heute. Herausforderungen - Transformationen - Impulse (in Vorbereitung).

Rahner, Karl: Grundkurs des Glaubens. Einführung in den Begriff des Christentums. Freiburg im Breisgau 1976.

Ruhstorfer, Karlheinz: Freiheit - Würde - Glauben. Christliche Religion und westliche Kultur, Paderborn 2015.

Stadlbauer, Helene: Klaus Heinrichs „Parmenides und Jona“: Kleine Archäologie menschlicher Bedrohungen und ihre theologischen-philosophischen Widerständigkeiten (DA), Wien 2014.

Vattimo, Gianni: Jenseits des Christentums. Gibt es eine Welt ohne Gott?, München / Wien 2004.

Vattimo, Gianni: Abschied. Theologie, Metaphysik und Philosophie heute, Wien 2003.

Vattimo, Gianni: „Os mé. Zur Haltung des ,als ob nicht“ bei Paulus und Heidegger“, in: Uhl, Florian / Boelderl, Artur R. (Hg.): Zwischen Verzückung und Verzweiflung. 
Dimensionen religiöser Erfahrung (Schriften der österreichischen Gesellschaft für Religionsphilosophie, Band 2), Düsseldorf 2001, 169-182.

Vattimo, Gianni: ,Dialektik, Differenz, schwaches Denken“, in: Schönherr-Mann, HansMartin (Hg.): Ethik des Denkens. Perspektiven von Ulrich Beck, Paul Ricoeur, Manfred Riedel, Gianni Vattimo, Wolfgang Welsch, München 2000, 79-97.

Vattimo, Gianni: Glauben - Philosophieren, Stuttgart 1997.

Vattimo, Gianni: Die transparente Gesellschaft, Wien 1992.

Wenzel, Knut: „Konkrete Globalität. Das Potential der Kirche zur Modellbildung für eine societas mundana“, in: Appel, Kurt / Deibl, Jakob (Hg.): Barmherzigkeit und zärtliche Liebe. Das theologische Programm von Papst Franziskus. Freiburg im Breisgau (erscheint im Juli 2016). 